\title{
Diffuse auroral scattering by whistler mode chorus waves: Dependence on wave normal angle distribution
}

\author{
Binbin Ni, ${ }^{1}$ Richard M. Thorne, ${ }^{1}$ Nigel P. Meredith, ${ }^{2}$ Yuri Y. Shprits, ${ }^{3,4}$ \\ and Richard B. Horne ${ }^{2}$ \\ Received 31 January 2011; revised 11 July 2011; accepted 15 July 2011; published 11 October 2011.
}

[1] Using the statistical CRRES measurements of the electric field intensities of lower band chorus (LBC) and upper band chorus (UBC) around $L=6$ under geomagnetically moderate conditions, we evaluate the variations in modeled magnetic field spectral intensity and the resultant changes in resonant scattering rates of plasma sheet electrons caused by different choices of the wave normal distribution. UBC scattering rates inferred from electric field measurements show a common trend of decreasing scattering with increasing peak wave normal angle, $\theta_{m}$, for the plasma sheet electrons at all resonant pitch angles. This trend is mainly due to the lower power of magnetic field as derived from the electric field measurements for oblique waves. The LBC resonant diffusion inferred from electric field measurements shows a considerable increase in scattering rates with increasing $\theta_{m}$ for $\sim 1 \mathrm{keV}$ electrons at all resonant pitch angles and for 3-30 keV electrons over certain ranges of pitch angles, which is contrary to the decrease in wave magnetic field amplitude and results mainly from the decrease in resonant energy and redistribution of the majority of wave power at large wave normal angles for increased peak wave normal angle. LBC-induced scattering rates of 3-10 keV electrons decrease with increasing $\theta_{m}$ at low pitch angles, consistent with the decrease in wave magnetic field amplitude when $\theta_{m}$ increases. Our investigation demonstrates that the knowledge of the wave normal distribution of LBC and UBC is essential for an accurate quantification of the net resonant scattering rates and loss timescales of the plasma sheet electrons for an improved global simulation of diffuse auroral precipitation and the evolution of plasma sheet electron pitch angle distribution if only measurements of wave electric field intensity are available. In contrast, the diffuse auroral scattering rates calculated from magnetic field measurements are much less sensitive to the assumption on wave normal angle distribution. While UBC scattering with constant magnetic field power is roughly insensitive to the assumed wave normal distribution, LBC scattering with constant magnetic field power becomes more dependent on the assumed wave normal angle distribution, especially for $\sim 1 \mathrm{keV}$ electrons.

Citation: Ni, B., R. M. Thorne, N. P. Meredith, Y. Y. Shprits, and R. B. Horne (2011), Diffuse auroral scattering by whistler mode chorus waves: Dependence on wave normal angle distribution, J. Geophys. Res., 116, A10207, doi:10.1029/2011JA016517.

\section{Introduction}

[2] Characteristically, whistler mode chorus waves occur in two frequency bands, a lower band $\left(f<0.5 f_{\text {ce }}\right)$ and an upper band $\left(0.5 f_{\mathrm{ce}}<f<f_{\mathrm{ce}}\right)$, where $f$ is the wave frequency

\footnotetext{
${ }^{1}$ Department of Atmospheric and Oceanic Sciences, University of California, Los Angeles, California, USA.

${ }^{2}$ British Antarctic Survey, Natural Environment Research Council, Cambridge, UK.

${ }^{3}$ Institute of Geophysics and Planetary Physics, University of California, Los Angeles, California, USA.

${ }^{4}$ Also at Department of Atmospheric and Oceanic Sciences, University of California, Los Angeles, California, USA.

Copyright 2011 by the American Geophysical Union. 0148-0227/11/2011JA016517
}

and $f_{\mathrm{ce}}$ is the equatorial electron gyrofrequency [Burtis and Helliwell, 1976; Meredith et al., 2001]. Although the mechanism for the generation of the gap at $0.5 f_{\text {ce }}$ remains unresolved, recent studies [Ni et al., 2008, 2011b; Su et al., 2009, 2010; Thorne et al., 2010; Tao et al., 2011a] have demonstrated that the two bands of nightside (00-06 MLT) chorus emissions play distinct roles in driving resonant scattering of the plasma sheet electrons with a pronounced dependence on both kinetic energy and pitch angle. Scattering by upper-band chorus (UBC) and lower-band chorus (LBC) acts in combination as the major contributor to the occurrence of the diffuse aurora and the formation of electron pancake distribution peaked at $90^{\circ}$ pitch angles in the inner magnetosphere. Resonant scattering by UBC is capable of precipitating $\sim 100 \mathrm{eV}$ to $\sim 2 \mathrm{keV}$ electrons on a 
timescale approaching the strong diffusion level [ $\mathrm{Ni}$ et al., 2008, 2011b; Thorne et al., 2010], and also accounts for the formation of the pancake distribution for $<\sim 2 \mathrm{keV}$ electrons. In contrast, $\mathrm{LBC}$ is most effective for precipitating $>2 \mathrm{keV}$ plasma sheet electrons. Net scattering by LBC and $\mathrm{UBC}$ on the nightside can cover a wide energy range from $\sim 100 \mathrm{eV}$ to $>100 \mathrm{keV}$ and a broad interval of equatorial pitch angle, thereby accounting for the enhanced electron anisotropy and the formation of electron pancake distribution that are frequently observed during the transport of electrons from the nightside to the dayside following their injection from the plasma sheet [Meredith et al., 1999, 2000; Li et al., 2010; Tao et al., 2011a].

[3] To quantitatively examine the chorus-driven scattering effects on the diffusive transport of plasma sheet electrons toward the loss cone and ultimate diffuse auroral precipitation into the atmosphere, quasi-linear diffusion coefficients need to be evaluated using a model of chorus waves described by a group of parameters that include the magnetic field wave amplitude, wave frequency spectrum, wave latitudinal distribution, and wave normal angle distribution [e.g., Lyons, 1974a, 1974b; Summers, 2005; Albert, 2005, 2007, 2008; Horne et al., 2005; Thorne et al., 2005, 2007, 2010; Summers et al., 2007a, 2007b; Summers and Ni, 2008; Shprits et al., 2007; Ni et al., 2008; Shprits and Ni, 2009; Su et al., 2009, 2010; Tao et al., 2011a]. While quasi-linear theory omits the nonlinear effects such as phase trapping by the wavefield, it has been well established and has been used successfully to provide an effective overall description of chorus-driven resonant diffusion at radiation belt energies by numerous publications. For instance, quasilinear scattering has been remarkably effective at quantifying both the rates of microburst scattering loss by chorus [Thorne et al., 2005] and the rates of local acceleration to relativistic energies [Horne et al., 2005; Thorne et al., 2007; Albert et al., 2009]. Albert [2010], using the two formulations of diffusion which are conceptually different, demonstrated that suitably averaging the monochromatic diffusion coefficients over chorus frequency and wave normal angle parameters can favorably reproduce the full broadband quasi-linear results. A recent study by Tao et al. [2011b] also found that a fully relativistic Lorentz test particle simulation of scattering induced by a broadband, incoherent, small-amplitude wavefield gives diffusion results in an excellent agreement with those derived from the quasi-linear theory of Kennel and Engelmann [1966]. These studies, together with numerous previous publications, have well justified the adoption of quasi-linear theory for this study which concentrates on the average scattering rates of plasma sheet electrons by statistically averaged chorus emissions.

[4] As an excellent database for plasma waves that contains a broad frequency range from $5.6 \mathrm{~Hz}$ to $400 \mathrm{kHz}$, a dynamic range covering a factor of $10^{5}$ in amplitude, and a good coverage of the low-latitude $\left(|\lambda|<\sim 25^{\circ}\right)$ radiation belt region [Anderson et al., 1992], the wave observations from the CRRES Plasma Wave Experiment have been extensively used to construct the global distribution and emission characteristics of chorus waves in the inner magnetosphere [Meredith et al., 2001, 2009], but unfortunately only measurements of electric field are available. As a consequence, wave normal angle information is required to convert the observed electric field data to magnetic field data for the quantification of scattering rates. An assumption of parallel propagation has been usually adopted to analyze the CRRES electric field wave data [e.g., Meredith et al., 2003; Ni et al., $2011 \mathrm{a}, 2011 \mathrm{~b}]$. There are a number of studies on the source and propagation properties of chorus emissions indicating that chorus propagates primarily parallel or quasi-parallel to the ambient magnetic field in the equatorial source region [e.g., Thorne and Kennel, 1967; Burton and Holzer, 1974; Goldstein and Tsurutani, 1984; Hospodarsky et al., 2001; Santolik et al., 2003], consistent with theoretical predictions [Kennel and Thorne, 1967; Bortnik et al., 2007]. However, direct or indirect observations of chorus wave normals in other cases suggested that the generation of chorus emissions, particularly at off-equatorial positions, occur with much higher wave normal angles with a dependence on the chorus band. For instance, using the wave measurements from Ogo 5 search coil magnetometer, Burton and Holzer [1974] reported a continuous variation of the wave normal distribution from $\leq 20^{\circ}$ near the equator to $\sim 90^{\circ}$ pointing away from the earth at higher latitudes for unducted chorus and a random distribution of wave normals within a cone of half angle $40^{\circ}$ from the ambient magnetic field for ducted chorus. Hayakawa et al. [1984] and Muto et al. [1987], using the GEOS 2 and GEOS 1 satellite observations respectively, showed that $\mathrm{UBC}$ can propagate very close to the local oblique resonance cone $\theta_{\text {res }}$ while LBC propagates at relatively small wave normal angles of $5^{\circ}-40^{\circ}$. Those two studies, by estimating the ratio between total electric and magnetic field intensities or by ray tracing simulations, also suggested that UBC is generated with its wave normal close to $\theta_{\text {res }}$ in the vicinity of the magnetic equator by an electrostatic instability. Using the Polar/PWI six-channel wave measurements, Lauben et al. [2002] showed that the chorus emission wave normal angle deduced at the source is systematically related to the frequency band, being $\sim 0^{\circ}$ for $\mathrm{UBC}$ and approximately the Gendrin angle $\theta_{G}[$ Gendrin, 1961] for LBC. Breneman et al. [2009] used the CLUSTER observations and reported that both $\mathrm{LBC}$ and UBC can be emitted in a broad spectrum of wave normal angles, i.e., LBC tends to be emitted near $\theta_{G}$ and at earthward pointing wave normal angles of between $-20^{\circ}$ and $-30^{\circ}$, while UBC is generally observed at higher wave normal angles between $20^{\circ}$ and $40^{\circ}$ (anti-earthward). Santolik et al. [2009] also analyzed in detail a nightside chorus event observed on board the CLUSTER spacecraft, showing that chorus emissions can be generated at highly oblique angles with respect to the terrestrial magnetic field. More recently, Haque et al. [2010], using the Polar data, found that UBC wave normal angles tend to remain at or rise toward $\theta_{\text {res }}$ for low and midlatitudes but move away from the resonance cone angle at higher latitudes due to strong Landau damping. In contrast, LBC emissions with wave normal angles $<20^{\circ}$ have the highest probability of occurrence in the latitude range of $10^{\circ}-50^{\circ}$, with a secondary occurrence peak in the range of $50^{\circ}-70^{\circ}$ in the off-equator latitude range of $10^{\circ}-25^{\circ}$.

[5] The unresolved controversy over the wave normal angle distribution of chorus emissions raises questions on the commonly adopted assumption of parallel or quasiparallel propagation for chorus waves and adds uncertainty to the appropriate rates of scattering. The differences in 
reported propagation characteristics for chorus emissions (both lower band and upper band) can introduce considerable changes in the conversion of CRRES observed electric field spectral densities to magnetic field spectral densities, which subsequently influences the quantification of the role of chorus scattering in driving diffuse auroral precipitation. In the present study we start with the statistically averaged electric field spectral densities of nightside chorus obtained from the CRRES observations. By selecting different peak wave normal angles for both LBC and UBC based on previous theoretical simulations and observations, we first compute the corresponding magnetic field spectral densities and use them to model the magnetic field amplitude and wave frequency spectrum for LBC and UBC. Evaluations of bounce-averaged quasi-linear diffusion coefficients are then carried out, using the UCLA Full Diffusion Code (FDC) $[\mathrm{Ni}$ et al., 2008; Shprits and Ni, 2009], to quantify the effects of chorus wave normal angle distribution on the quantitative contributions of chorus emissions to diffuse auroral precipitation.

\section{CRRES Wave Data and Analysis}

[6] Here we use the statistical electric field spectral density data under geomagnetically moderate conditions for LBC and UBC, based on the CRRES observations within the 00-06 MLT sector where the activities for both the waves [e.g., Meredith et al., 2001, 2009; Li et al., 2009b] and the diffuse aurora [e.g., Petrinec et al., 1999; Anderson et al., 2001; Newell et al., 2009, 2010] are most intense. The electric field spectral densities are analyzed respectively within three specified magnetic latitude intervals $\left(|\lambda| \leq 5^{\circ}\right.$, $5^{\circ}<|\lambda| \leq 10^{\circ}$, and $\left.10^{\circ}<|\lambda| \leq 15^{\circ}\right)$ for LBC and two latitude intervals $\left(|\lambda| \leq 5^{\circ}\right.$ and $\left.5^{\circ}<|\lambda| \leq 10^{\circ}\right)$ for $\mathrm{UBC}$, in steps of $0.1 f_{c e}$ and $0.1 \mathrm{~L}$. The averaged electric field spectral densities $I_{E}$ at $L=5.9-6.1$, shown in the top panels of Figures $1 \mathrm{a}-1 \mathrm{c}$ for LBC and of Figures $1 \mathrm{~d}-1 \mathrm{e}$ for UBC, are utilized to construct a model of the chorus magnetic spectral intensity at $L=6$ (bottom panels of Figures 1a-1e) at each of the specified magnetic latitude interval, as explained below.

[7] To convert electric field spectral density $I_{E}$ to magnetic field spectral density $I_{B}$, we apply the Faraday Law,

$$
I_{B}=I_{E}\left(\frac{n}{c}\right)^{2} \sin ^{2} \beta
$$

where $n$ is the refractive index, $c$ is the speed of light, and $\beta$ is the angle between the wave electric field $\vec{E}$ and the wave propagation vector $\vec{k}$. The full cold-plasma dispersion relation for whistler mode waves is used to compute the refractive index $n$ is determined from the dispersion matrix and the Maxwell's equations by

$$
\beta=\arccos \left[\frac{\sin \theta\left(n^{2}-P\right)}{\sqrt{\left(n^{2} \sin ^{2} \theta-P\right)^{2}+\frac{D^{2}\left(n^{2} \sin ^{2} \theta-P\right)^{2}}{\left(n^{2}-S\right)^{2}}+n^{4} \sin ^{2} \theta \cos ^{2} \theta}}\right] .
$$

[8] The variation of $\beta$ as well as $n$ with wave frequency normalized to $f_{c e}$ at $L=6$ is illustrated in Figure 2: Figures $2 \mathrm{a}-2 \mathrm{~b}$ for $\mathrm{LBC}$ with four representative peak wave normal angles $\theta_{m}=0^{\circ}, 20^{\circ}, 40^{\circ}$ and $55^{\circ}$, and Figures $2 \mathrm{c}-2 \mathrm{~d}$ for UBC with $\theta_{m}=0^{\circ}, 20^{\circ}, 40^{\circ}$ and $44^{\circ}$. These peak wave normal angles for LBC and UBC are selected, based on the previous theoretical simulations and observations [e.g., Thorne and Kennel, 1967; Burton and Holzer, 1974; Goldstein and Tsurutani, 1984; Hayakawa et al., 1984; Muto et al., 1987; LeDocq et al., 1998; Hospodarsky et al., 2001; Lauben et al., 2002; Bortnik et al., 2007; Li et al., 2009b; Breneman et al., 2009; Santolik et al., 2003, 2009; Haque et al., 2010], to perform a parametric study and to investigate the sensitivity of chorus-driven diffuse auroral scattering to wave normal angle distribution. The ratio of plasma frequency to electron gyrofrequency is set as 6.4 based on the CRRES observations. For both LBC and UBC, the refractive index increases with increasing wave normal angle at any fixed normalized wave frequency. For parallel propagation $\vec{E}$ remains perpendicular to $\vec{k}\left(\beta=90^{\circ}\right)$ regardless of wave frequency. When chorus waves are oblique, $\beta$ decreases dramatically with increasing wave normal angle and normalized wave frequency. Moreover, the refractive index for UBC increases monotonously with normalized wave frequency more rapidly at larger wave normal angle. The increase in $n$ is most pronounced when the wave normal angle approaches $\theta_{\text {res }}$, where $\vec{E}$ and $\vec{k}$ become parallel and the waves become electrostatic.

[9] Based on equations (1)-(3), the magnetic field spectral densities (Figures 1a-1e, bottom panels, dotted curves) are obtained from the observed CRRES electric field spectral densities (Figures 1a-1e, top panels). $I_{B}$ of both LBC and UBC show a pronounced decrease for increasing wave normal angle, due to a combined effect of the increase in $n$ and the decreases in $\beta$. A least squares Gaussian fit (Figures 1a-1e, bottom panels, solid curves) is applied to each converted $I_{B}$ by assuming a frequency distribution given by [e.g., Lyons, 1974a, 1974b; Glauert and Horne, 2005; Ni et al., 2008]

$$
n^{2}=\frac{R L \sin ^{2} \theta+P S\left(1+\cos ^{2} \theta\right)-\sqrt{\left[R L \sin ^{2} \theta+P S\left(1+\cos ^{2} \theta\right)\right]^{2}-4 P R L\left(S \sin ^{2} \theta+P \cos ^{2} \theta\right)}}{2\left(S \sin ^{2} \theta+P \cos ^{2} \theta\right)}
$$

where $R, L, P$, and $S$ are the Stix parameters [Stix, 1962] and $\theta$ is the wave normal angle. The angle between $\vec{E}$ and $\vec{k}, \beta$,

$$
I_{B}(f)=A \exp \left[-\left(\frac{f-f_{m}}{\Delta f}\right)^{2}\right],\left(f_{l c}<f<f_{u c}\right)
$$


Lower-band chorus $\left(0.05<\mathrm{f} / \mathrm{f}_{\mathrm{ce}}<0.5\right)$

(a) $|\lambda| \leq 5^{\circ}$
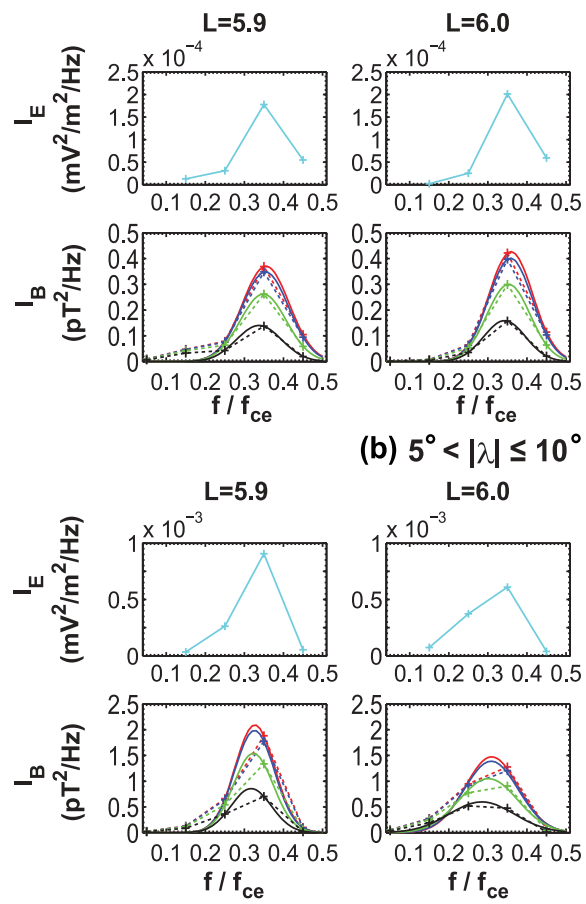

(b) $5^{\circ}<|\lambda| \leq 10^{\circ}$
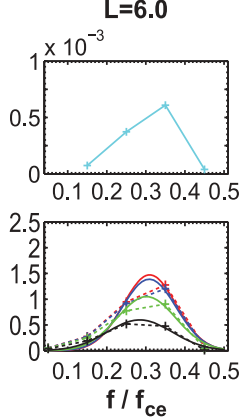

(c) $10^{\circ}<|\lambda| \leq 15^{\circ}$
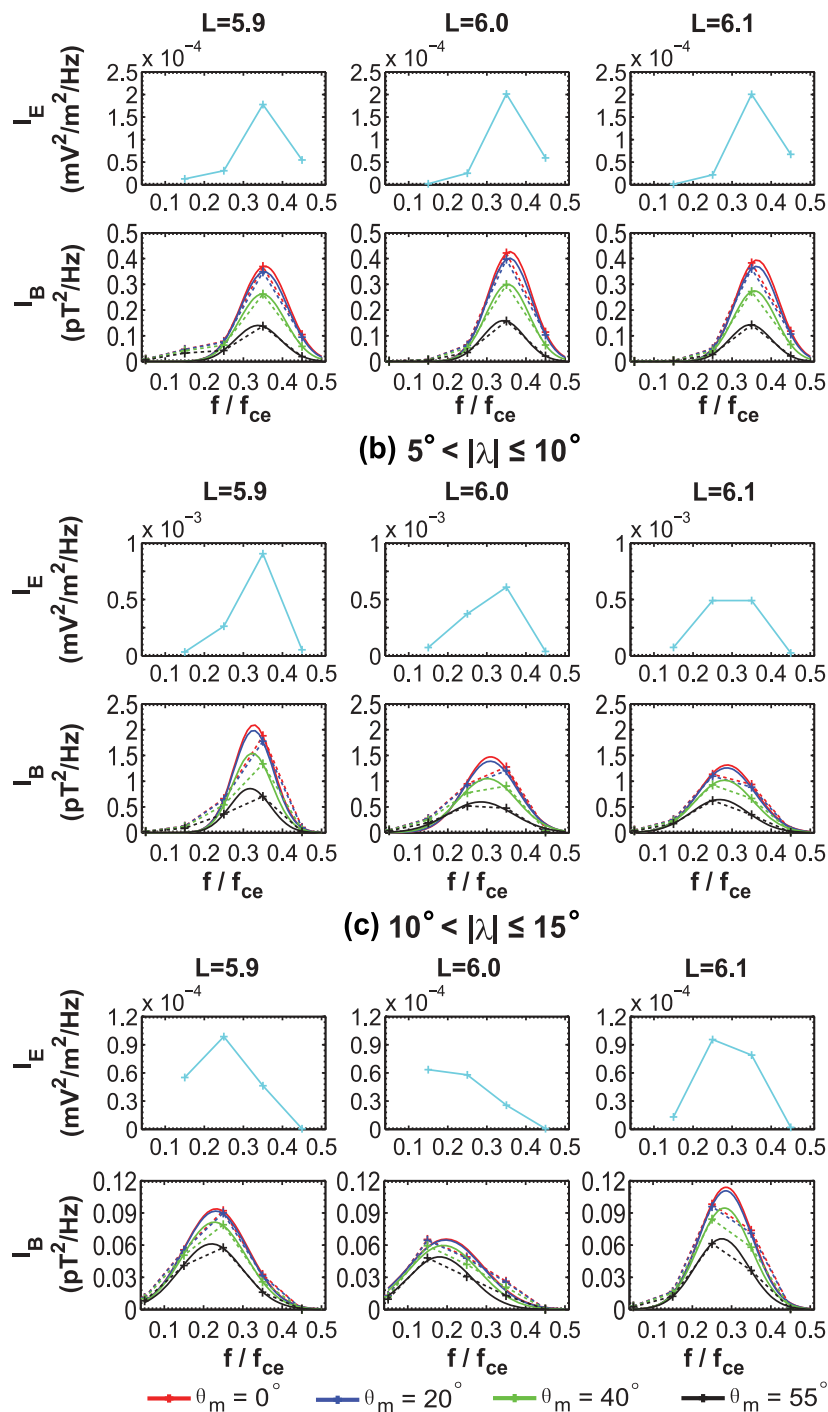

Upper-band chorus $\left(0.5<f / f_{c e}<0.7\right)$

(d) $|\lambda| \leq 5^{\circ}$

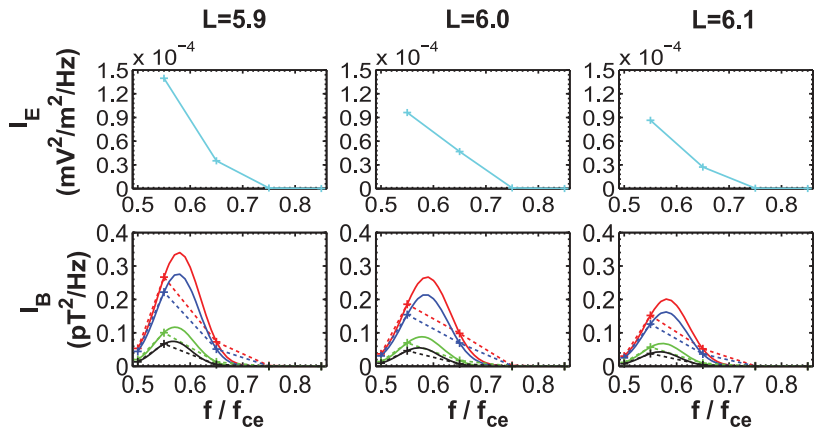

(e) $5^{\circ}<|\lambda| \leq 10^{\circ}$

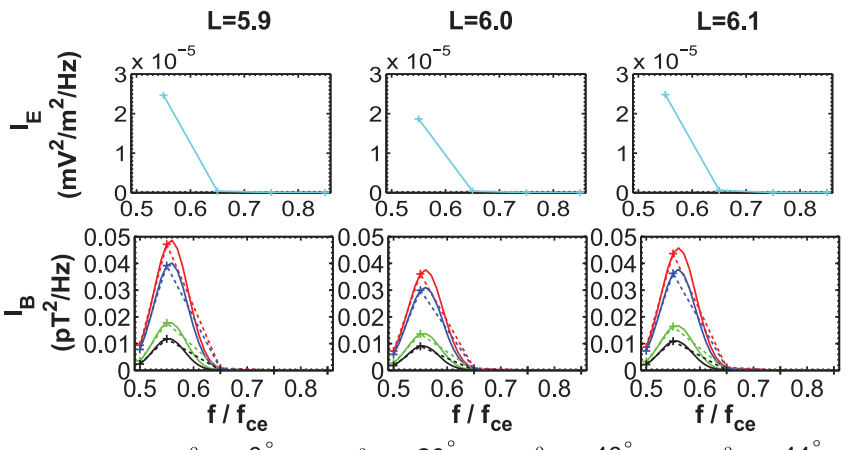

Figure 1. Averaged electric field spectral densities $I_{E}$ (top panels) at $L=5.9-6.1$ for lower band chorus within three magnetic latitude intervals of (a) $|\lambda| \leq 5^{\circ}$, (b) $5^{\circ}<|\lambda| \leq 10^{\circ}$, and (c) $10^{\circ}<|\lambda| \leq 15^{\circ}$, and for upper band chorus within two magnetic latitude intervals of (d) $|\lambda| \leq 5^{\circ}$ and (e) $5^{\circ}<|\lambda| \leq 10^{\circ}$, based on the CRRES wave observations under geomagnetically moderate conditions. Converted magnetic field spectral densities $I_{B}$ are shown in the bottom panels as dotted curves, corresponding to the indicated color-coded peak wave normal angles $\theta_{m}$. The modeled Gaussian fits are shown as solid curves.

where $f_{m}$ and $\Delta f$ are the frequency of the maximum wave power and the bandwidth, respectively, $f_{l c}\left(0.05 f_{c e}\right.$ for $\mathrm{LBC}$ and $0.5 f_{c e}$ for $\left.\mathrm{UBC}\right)$ and $f_{u c}\left(0.5 f_{c e}\right.$ for $\mathrm{LBC}$ and $0.7 f_{c e}$ for UBC) are the lower and upper cutoffs to the wave spectrum outside which the wave power is assumed to be zero, and $A$ is a normalization factor given by

$$
A=\frac{B_{w}^{2}}{\Delta f} \frac{2}{\pi^{1 / 2}}\left[\operatorname{erf}\left(\frac{f_{m}-f_{l c}}{\Delta f}\right)+\operatorname{erf}\left(\frac{f_{u c}-f_{m}}{\Delta f}\right)\right]^{-1},
$$

where $B_{w}$ is the wave magnetic field amplitude and erf is the error function. The 3-min averaged CRRES wave data of use gives us the wave spectral intensities at eight fre- quencies from $0.15 f_{c e}$ to $0.85 f_{c e}$ with an increment of $0.1 f_{c e}$. For LBC, there are four points (from $0.15 f_{c e}$ to $0.45 f_{c e}$ ) available. To apply the Gaussian fit, we artificially add another point at $0.05 f_{c e}$ with the wave spectral intensity equal to a tenth of the wave spectral intensity at $0.15 f_{c e}$ based on the knowledge that LBC wave power is very weak at very low frequencies. For UBC, there are only three points (from $0.55 f_{c e}$ to $0.85 f_{c e}$ ) available. Because characteristically there is a gap in wave intensity at half the electron gyrofrequency, we artificially add another point at $0.5 f_{c e}$ with the wave spectral intensity being a tenth of the wave spectral intensity at $0.55 f_{c e}$ to apply the Gaussian fit to the UBC spectrum. We note that for the Gaussian fits to 
Lower-band chorus $\left(0.05<\mathrm{f} / \mathrm{f}_{\mathrm{ce}}<0.5\right)$

(a) Refractive index

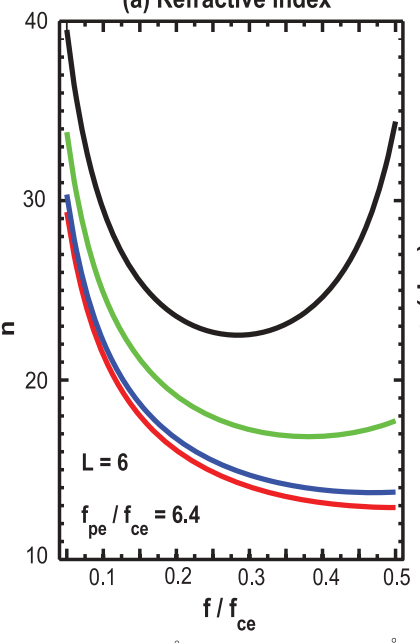

(b) Angle between $\mathrm{E}$ and $\mathrm{k}$

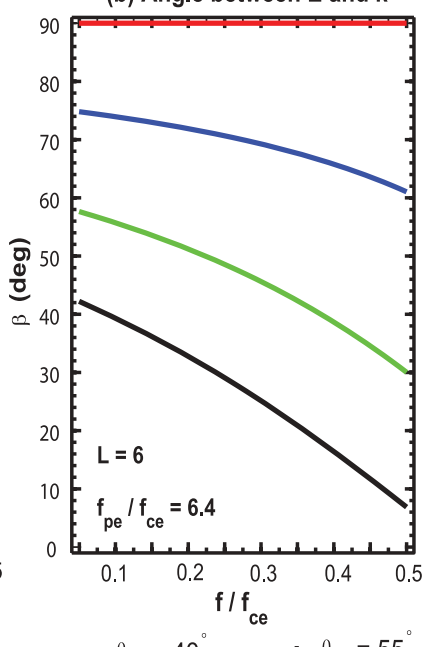

$\rightarrow \theta_{\mathrm{m}}=40^{\circ} \quad \rightarrow{ }_{\mathrm{m}}=55^{\circ}$

Upper-band chorus $\left(0.5<f / f_{c e}<0.7\right)$
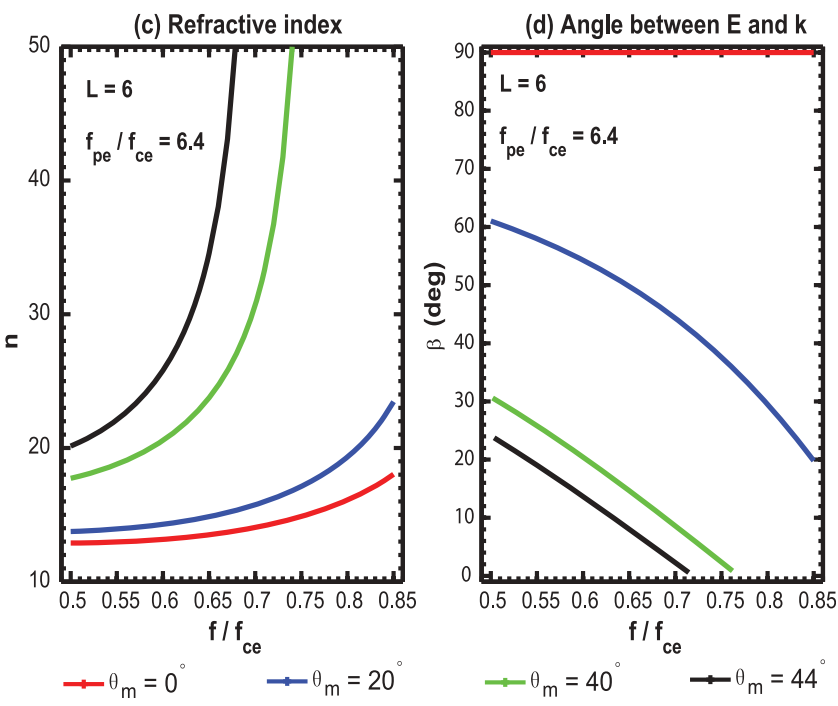

Figure 2. Variations of refractive index $(n)$ and angle between the wave electric field and propagation vector $(\beta)$ with normalized wave frequency for ( $a$ and $b$ ) lower band chorus and for (c and d) upper band chorus at $L=6$, corresponding to the indicated color-coded peak wave normal angles $\theta_{m}$.

LBC and UBC spectrum the above treatments follow the characteristic features of chorus measurements [e.g., Burtis and Helliwell, 1976; Meredith et al., 2001] but the choice of a tenth value is rather arbitrary. We have checked the other choices of the relative values $(\leq 0.2)$ at the artificial frequency points and found that their effects are minor since the wave power within $0.05-0.15 f_{c e}$ and $0.5-0.55 f_{c e}$ is relatively small compared to the rest of LBC and UBC wave power. Subsequently, parameters for the Gaussian fits, including the magnetic field amplitude $\left(B_{w}\right)$, normalized peak frequency $\left(\overline{f_{m}}=f_{m} / f_{c e}\right)$, and normalized bandwidth $\left(\overline{\Delta f}=\Delta f / f_{c e}\right)$ for LBC and UBC, are averaged over $L=$ 5.9-6.1 and listed in Table 1 for the different choices of peak wave normal angle $\theta_{m}$. Compared to the parallel propagation case $\left(\theta_{m}=0^{\circ}\right)$, the changes in the modeled wave frequency distribution are small for both $\mathrm{LBC}$ and UBC. However, the modeled average UBC wave amplitudes for $\theta_{m}=44^{\circ}$ are lower than for the field-aligned case by a factor of 2, while the decrease of LBC wave amplitude with increasing $\theta_{m}$ is relatively smaller, by a factor of 1.2-1.7. This difference between UBC and LBC is mainly due to a slower decrease in $\beta$ with $\theta_{m}$ for LBC.

\section{Resonant Diffusion Rates of Plasma Sheet Electrons}

[10] Our computation of bounce-averaged quasi-linear scattering rates includes the contributions from the cyclotron harmonic resonances between $\mathrm{N}=-5$ and $\mathrm{N}=5$ and the Landau resonance $\mathrm{N}=0$, using the UCLA Full Diffusion Code (FDC) [Ni et al., 2008; Shprits and Ni, 2009]. The full cold plasma dispersion relation for a hydrogen plasma is adopted in combination with the relativistic Doppler-shifted resonance condition to determine the resonant frequencies for each resonance harmonic, which are subsequently input into the bounce-averaged quasi-linear diffusion coefficient formulae [e.g., Glauert and Horne, 2005; Albert, 2007] to calculate the net scattering rates. The equatorial electron number density and magnetic field at $L=6$ are taken to be $6.5 \mathrm{~cm}^{-3}$ and $127.5 \mathrm{nT}$, respectively, based on the CRRES moderate-time observations. The wave normal distribution of chorus wave power is assumed to be Gaussian, given by

$$
g(\theta)=\exp \left[-\left(\frac{\tan \theta-\tan \theta_{m}}{\tan \theta_{w}}\right)^{2}\right]\left(\theta_{l c} \leq \theta \leq \theta_{u c}\right),
$$

where $\theta_{m}$ is the peak wave normal angle with maximum wave power, $\theta_{w}$ the angular width, and $\theta_{l c}$ and $\theta_{u c}$ the lower and upper bounds to the wave normal distribution outside which the wave power is zero. In the present study we fix the values of $\theta_{l c}\left(=0^{\circ}\right.$ for both LBC and UBC $), \theta_{u c}\left(=58^{\circ}\right.$ for $\mathrm{LBC}$ and $44^{\circ}$ for $\left.\mathrm{UBC}\right)$, and $\theta_{w}\left(=30^{\circ}\right.$ for both $\mathrm{LBC}$ and $\mathrm{UBC}$ ) but vary the value of $\theta_{m}$ to be consistent with the choice of modeled wave power frequency spectrum. The wave power distribution over frequency and wave normal angle is assumed to be constant within each of the specified magnetic latitude intervals. We also assume that the electron density is constant with magnetic latitude and that the magnetic field is dipolar, scaled by the CRRES observed equatorial magnetic field strength. Readers are referred to a number of previous studies [Lyons, 1974a, 1974b; Schulz and Lanzerotti, 1974; Glauert and Horne, 2005; Albert, 2005, 2007, 2008; Shprits et al., 2006a; Summers et al., 2007a] for the formulation of quasi-linear diffusion coefficients in a dipole magnetic field.

[11] Figure 3 shows bounce-averaged pitch angle diffusion rates $\left\langle D_{\alpha \alpha}\right\rangle$, momentum diffusion rates $\left\langle D_{p p}\right\rangle$, and mixed (pitch angle, momentum) diffusion rates $\left\langle D_{\alpha p}\right\rangle$ as a function of equatorial pitch angle $\alpha_{e q}$ and electron kinetic energy $E_{k}$ at $L=6$ for electrons between $100 \mathrm{eV}$ and $100 \mathrm{keV}$ due to resonant interactions with $\mathrm{LBC}$ at the four adopted peak wave normal angles. Pronounced decreases in bounceaveraged diffusion rates for electrons above a few $\mathrm{keV}$ are apparent when $\theta_{m}$ varies from $0^{\circ}$ to $55^{\circ}$. The loss timescales of these electrons, approximated by the inversion of $\left\langle D_{\alpha \alpha}\right\rangle$ at the edge of equatorial loss cone [e.g., Shprits et al., 2006b; 
Table 1. Magnetic Field Amplitude $B_{w}$, Peak Wave Frequency $f_{m}$, and Bandwidth $\Delta f$ Obtained by Applying Gaussian Fits to Magnetic Field Intensities Converted From CRRES Observed Averaged Electric Field Intensities Over $L=5.9-6.1$ in the Specified Magnetic Latitude Intervals for Nightside (00:00-06:00 MLT) Lower Band and Upper Band Chorus Under Geomagnetically Moderate Conditions $\left(100 \mathrm{nT}^{2} \mathrm{AE}^{*}<300 \mathrm{nT}\right)^{\mathrm{a}}$

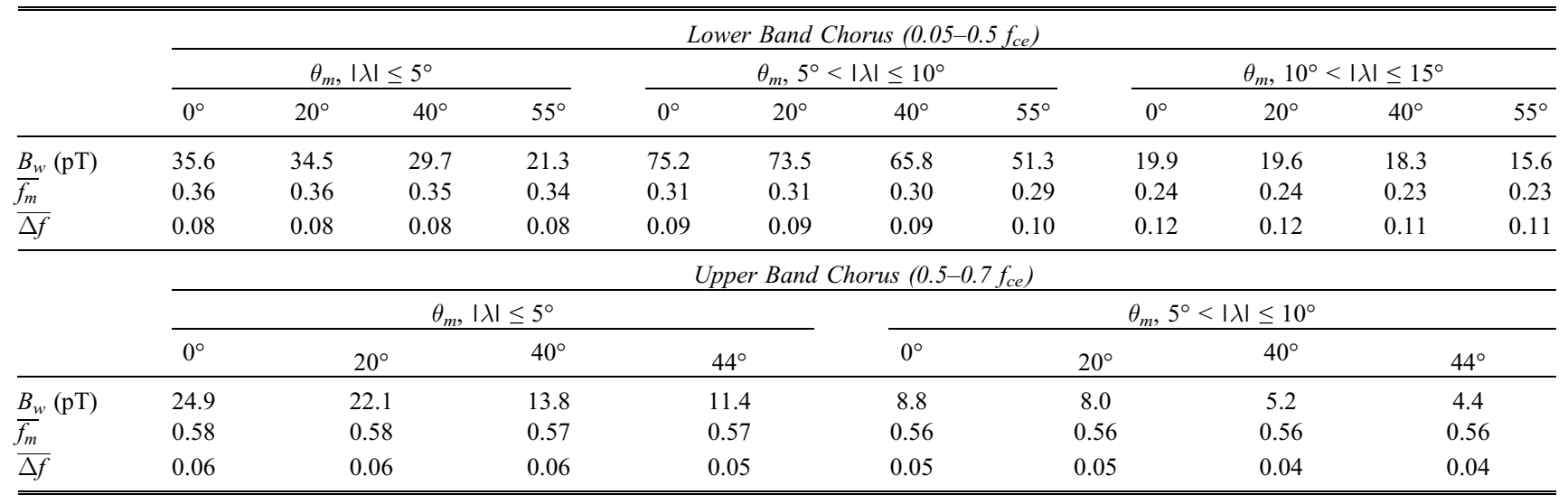

${ }^{\mathrm{a}}$ Four choices of peak wave normal angle $\theta_{m}$ are considered for lower band chorus and upper band chorus.

Summers et al., 2007a; Albert and Shprits, 2009], change from less than one hour for the case of $\theta_{m}=0^{\circ}$ and $20^{\circ}$ to several hours for $\theta_{m}=55^{\circ}$. Pitch angle scattering by more oblique LBC can occur for electrons $<\sim 1 \mathrm{keV}$, while electrons at these energies are out of resonance for scattering by quasi-parallel propagation $\left(\theta_{m}=0^{\circ}\right)$ LBC. In addition, both momentum diffusion $\left\langle D_{p p}\right\rangle$ and mixed diffusion $\left|\left\langle D_{\alpha p}\right\rangle\right|$ show a tendency to increase for $\sim 1-2 \mathrm{keV}$ electrons (mainly due to the Laudan resonance) and a tendency to decrease for higher energy electrons when LBC emissions become more oblique.

[12] A more detailed comparison of LBC scattering rates with respect to the four wave normal angle distributions is presented in Figure 4 for four specified energies from $1 \mathrm{keV}$ to $30 \mathrm{keV}$. In Figure $4\left\langle D_{\alpha \alpha}\right\rangle$ is also compared to the strong diffusion rates $D_{s d}$, computed using equation (27) of

\section{Lower-band chorus $\left(0.05<f / f_{c e}<0.5\right)$}

(a) $\theta_{m}=0^{\circ}$
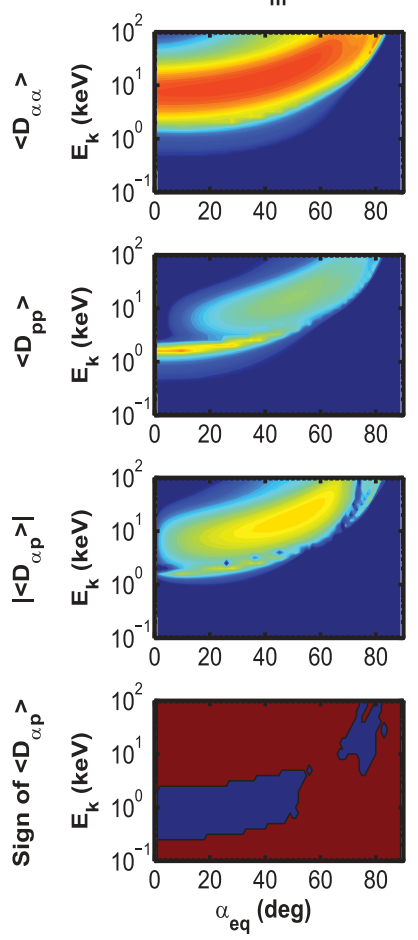

(b) $\theta_{m}=20^{\circ}$
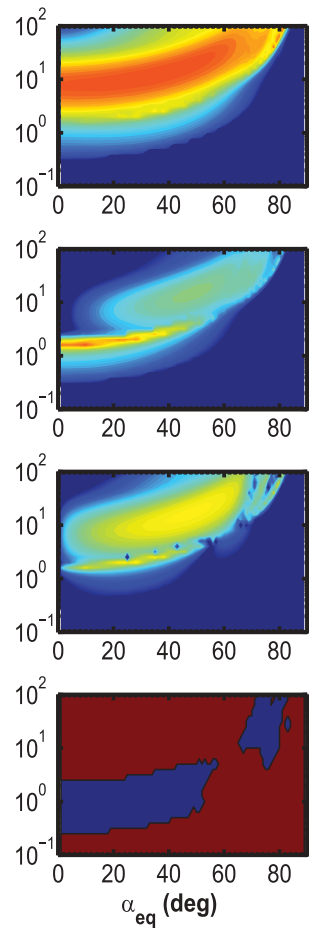

(c) $\theta_{m}=40^{\circ}$
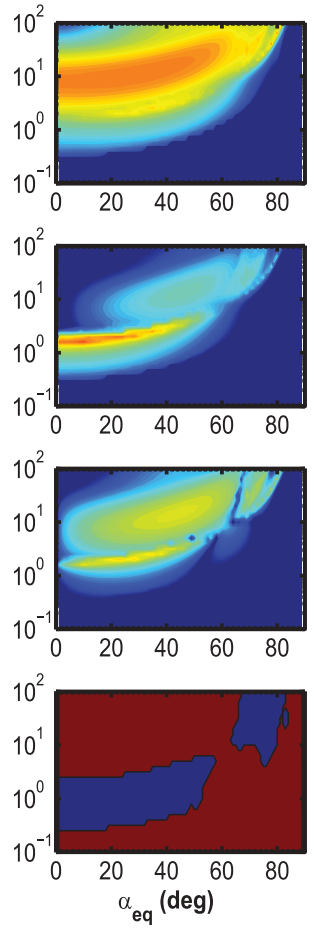

(d) $\theta_{m}=55^{\circ}$
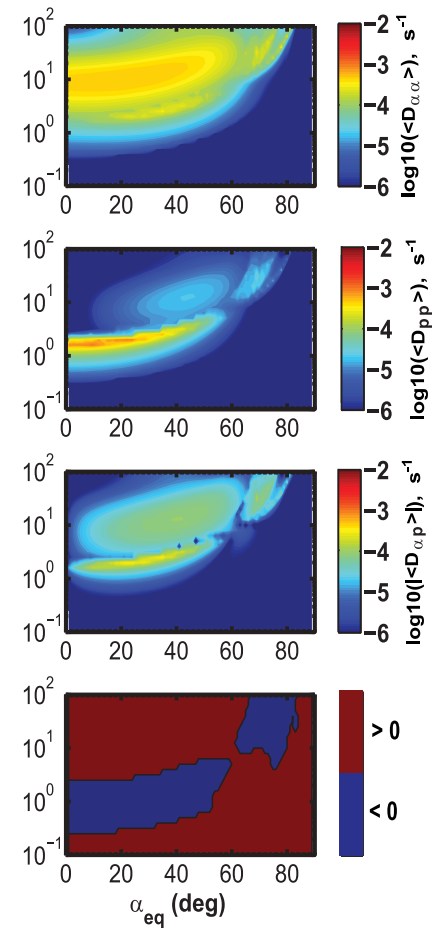

Figure 3. Bounce-averaged diffusion coefficients at $L=6$ (top to bottom, $\left\langle D_{\alpha \alpha}\right\rangle,\left\langle D_{p p}\right\rangle,\left|\left\langle D_{\alpha p}\right\rangle\right|$ and sign of $\left.\left\langle D_{\alpha p}\right\rangle\right)$ as a function of equatorial pitch angle $\alpha_{e q}$ and electron kinetic energy $E_{k}$ for moderate-time lower band chorus corresponding to four wave normal angle distributions with peak wave normal angle $\theta_{m}$ at (a) $0^{\circ}$, (b) $20^{\circ}$, (c) $40^{\circ}$, and (d) $55^{\circ}$. 


\section{Lower-band chorus $\left(0.05<\mathrm{f} / \mathrm{f}_{\mathrm{ce}}<0.5\right)$}

(a) $1 \mathrm{keV}$
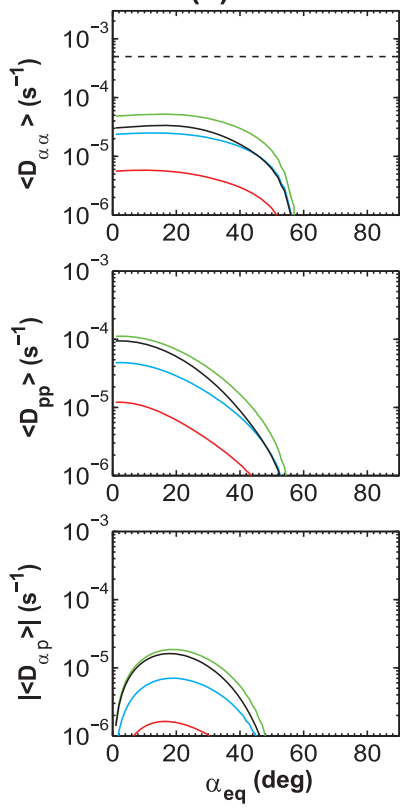

$\rightarrow \theta_{m}=0$ (b) $3 \mathrm{keV}$
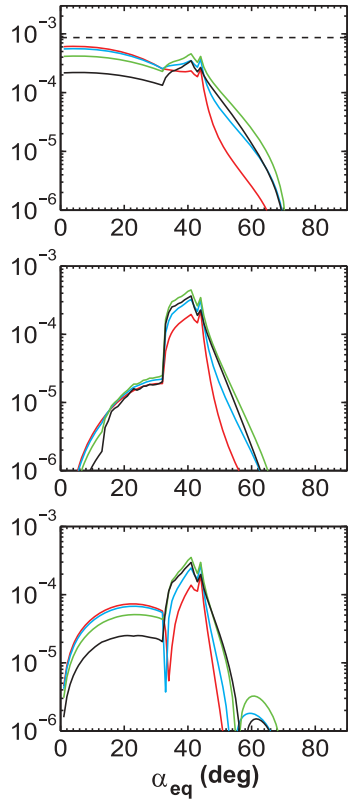

(c) $10 \mathrm{keV}$
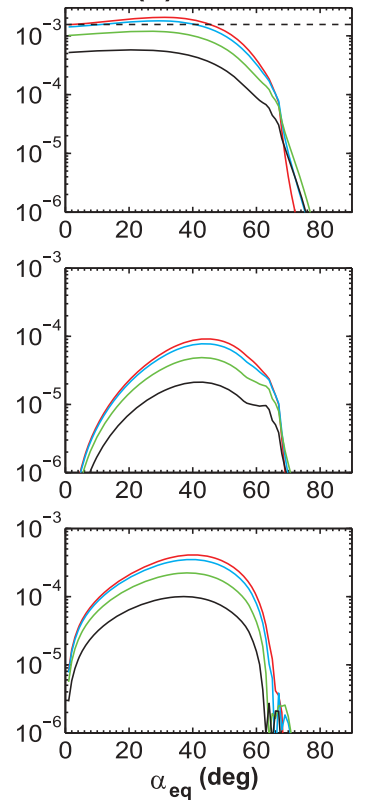

$\longrightarrow \theta_{\mathrm{m}}=40^{\circ} \quad{ }^{\theta_{m}}=55$ (d) $30 \mathrm{keV}$
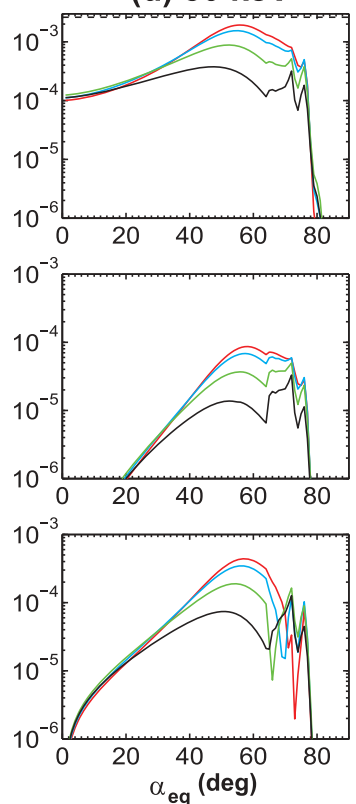

Figure 4. Comparison of lower band chorus induced bounce-averaged resonant diffusion coefficients at $L=6$ as a function of equatorial pitch angle for plasma sheet electrons at the five specified energies from $500 \mathrm{eV}$ to $10 \mathrm{keV}$ by adopting the four wave normal angle distributions peaking respectively at $0^{\circ}, 20^{\circ}$, $40^{\circ}$, and $55^{\circ}$. The horizontal dashed lines represent the strong diffusion rate $D_{S D}$.

Summers and Thorne [2003] and shown as horizontal dashed lines. Interestingly, for $1 \mathrm{keV}$ electrons, all three diffusion rates increase with $\theta_{m}$ up to $\theta_{m}=\sim 40^{\circ}$, despite the decrease in wave magnetic field amplitude. For $\theta_{m}=55^{\circ}$, the scattering rates are smaller than those for $\theta_{m}=40^{\circ}$ but still much larger than those for $\theta_{m}=0^{\circ}$. Therefore, variations in wave normal angle distribution are solely responsible for the increases in LBC scattering rates for $1 \mathrm{keV}$ electrons. Two factors contribute to this increase. One factor is that at fixed wave frequency the minimum resonant electron energy decreases for chorus with increased wave normal angle. As a consequence, while $1 \mathrm{keV}$ electrons are just slightly too low in energy to resonate with parallel propagating $\mathrm{LBC}$, increasing the wave normal angle can bring them into resonance with oblique LBC emissions. The other factor is that with larger $\theta_{m}$ more wave power is distributed at intermediate and high wave normal angles so that $1 \mathrm{keV}$ electrons can be subject to efficient scattering by oblique LBC emissions even though $B_{w}$ is weaker than the quasiparallel propagation case. This effect of LBC scattering only occurs for a limited range of energies near $1 \mathrm{keV}$. For 3 and $10 \mathrm{keV}$ electrons, the scattering rates at lower $\alpha_{e q}\left(<\sim 30^{\circ}\right.$ for $3 \mathrm{keV}$ and $<\sim 60^{\circ}$ for $10 \mathrm{keV}$ ) decrease with increasing $\theta_{m}$, due to a combined effect of decrease in wave magnetic field amplitude and variation of wave power distribution over wave normal angle. To differentiate between these two effects we compute the bounce-averaged diffusion coefficients for the four different wave normal angle distributions for a fixed $B_{w}$, which we set to the value for $\theta_{m}=0^{\circ}$. The results are displayed in Figure 5 and show that there are noticeable changes in diffusion rates over the range $30^{\circ}-70^{\circ}$ for $3 \mathrm{keV}$ electrons and over $0^{\circ}-70^{\circ}$ for 10 and $30 \mathrm{keV}$ electrons, which are clearly associated with the wave normal angle model and are unrelated to the wave amplitude. Furthermore, use of the larger wave normal angle models tends to extend LBC scattering closer to $\alpha_{e q}=90^{\circ}$, especially for 3 and $10 \mathrm{keV}$ electrons, which further demonstrates that the wave power distribution over wave normal angle plays an important role in the accurate evaluation of LBC-driven diffuse auroral scattering rates. On the other hand, with a fixed $B_{w}$, the differences in the scattering rates between the four wave normal models for $3-30 \mathrm{keV}$ electrons are greatly reduced, suggesting that the wave magnetic field amplitude is the dominant factor controlling the resonant scattering of $>3 \mathrm{keV}$ electrons by LBC. As a consequence, given the electric field intensities, quasi-parallel propagating LBC can cause the scattering loss of these electrons approaching the strong diffusion limit while highly oblique LBC results in lower scattering rates.

[13] Bounce-averaged diffusion rates $\left(\left\langle D_{\alpha \alpha}\right\rangle,\left\langle D_{p p}\right\rangle\right.$, and $\left.\left\langle D_{\alpha p}\right\rangle\right)$ for $100 \mathrm{eV}-100 \mathrm{keV}$ electrons due to UBC scattering at $\mathrm{L}=6$ are presented in Figure 6, corresponding to four UBC wave normal angle distributions with $\theta_{m}=0^{\circ}, 20^{\circ}, 40^{\circ}$ and $44^{\circ}$, respectively. Considerable decreases occur for all three diffusion coefficients when $\theta_{m}$ varies from $0^{\circ}$ to $44^{\circ}$. For the cases with $\theta_{m}=0^{\circ}$ and $20^{\circ}, \mathrm{UBC}$ can cause very efficient pitch angle scattering (on a timescale of hours or less near the loss cone) of plasma sheet electrons from $100 \mathrm{eV}$ to $\sim 2 \mathrm{keV}$, covering a broad range of $\alpha_{e q}$ from the loss cone to high angles. In contrast, for highly oblique UBC propagation, the diffusion rates decrease substantially for all considered energies and pitch angles. Efficient pitch angle 


\section{Lower-band chorus $\left(0.05<\mathrm{f} / \mathrm{f}_{\mathrm{ce}}<0.5\right)$}

(a) $1 \mathrm{keV}$
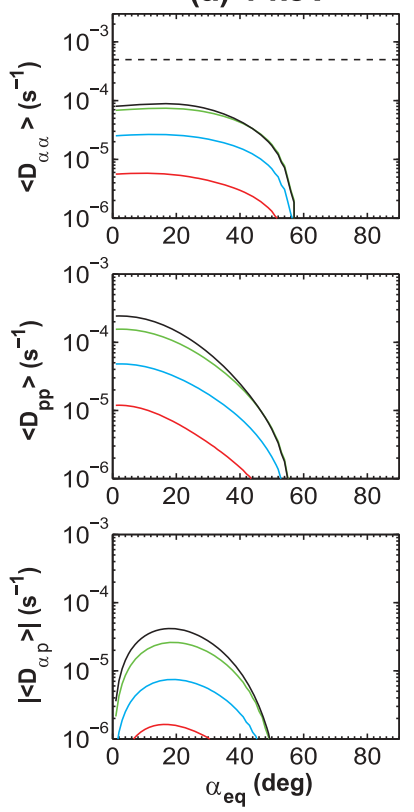

$+\theta_{m}=0$ (b) $3 \mathrm{keV}$
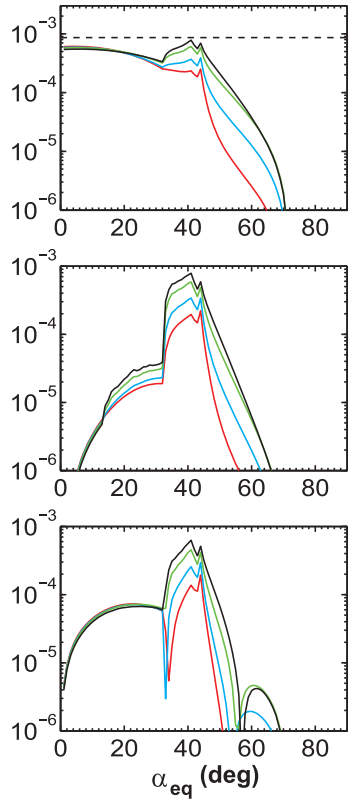

$\rightarrow \theta_{m}=20^{\circ}$ (c) $10 \mathrm{keV}$
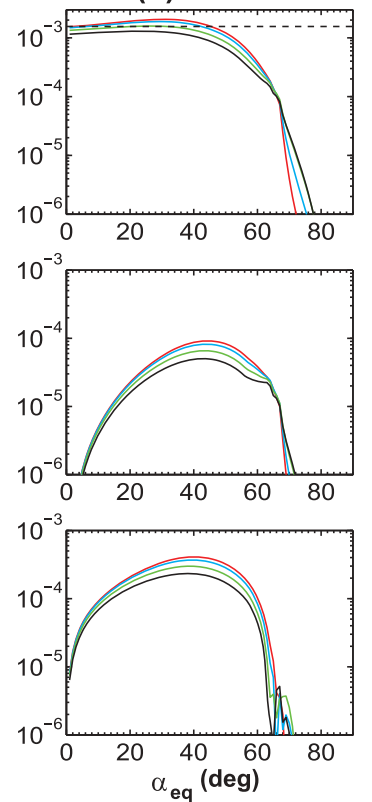

$\longrightarrow \theta_{\mathrm{m}}=40^{\circ} \quad \rightarrow \theta_{\mathrm{m}}=55$ (d) $30 \mathrm{keV}$
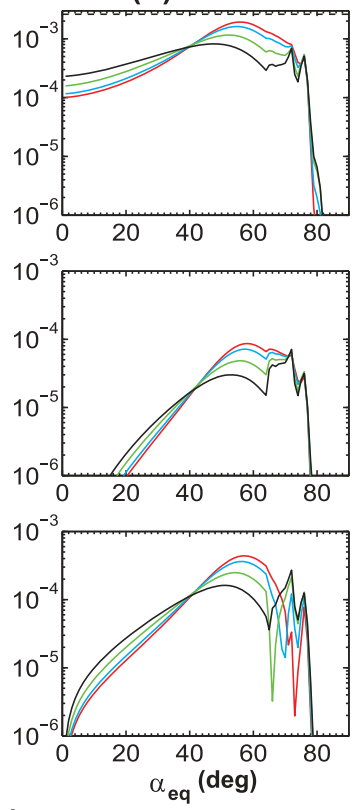

Figure 5. Same as in Figure 4, except that the adopted lower band chorus magnetic field amplitude is the same as that for $\theta_{m}=0^{\circ}$.

Upper-band chorus $\left(0.5<\mathrm{f} / \mathrm{f}_{\text {ce }}<0.7\right)$

(a) $\theta_{m}=0^{\circ}$
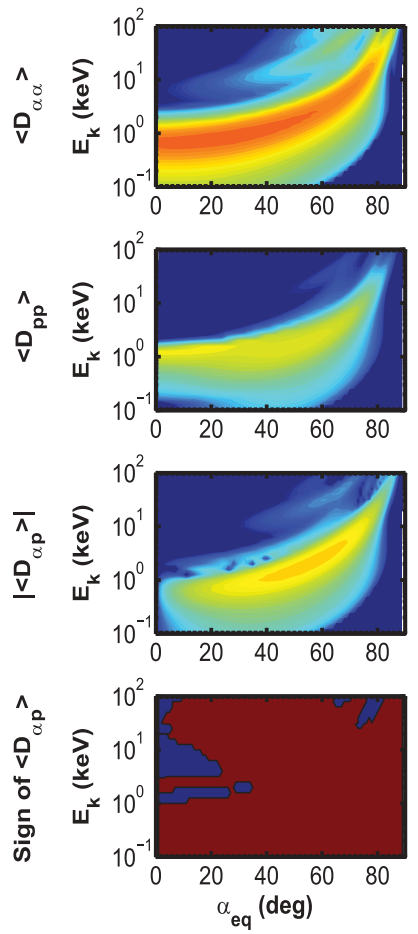

(b) $\theta_{m}=20^{\circ}$
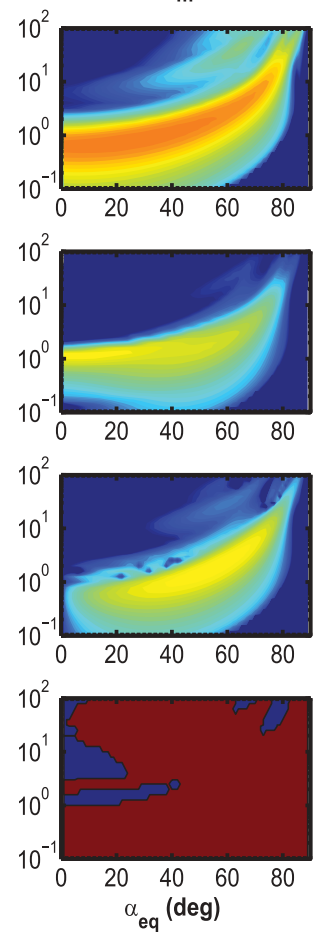

(c) $\theta_{m}=40^{\circ}$
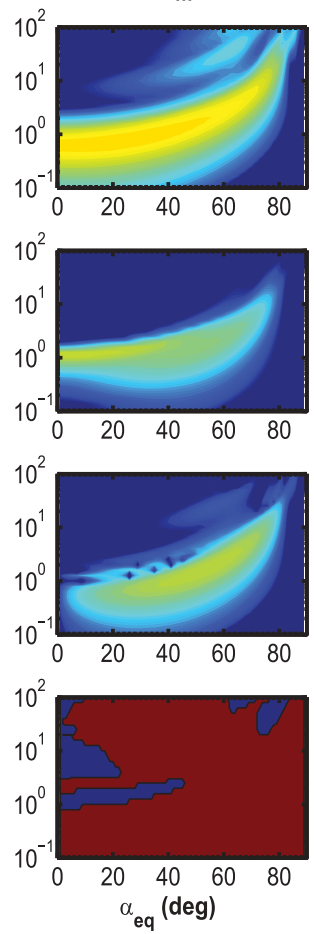

(d) $\theta_{m}=44^{\circ}$
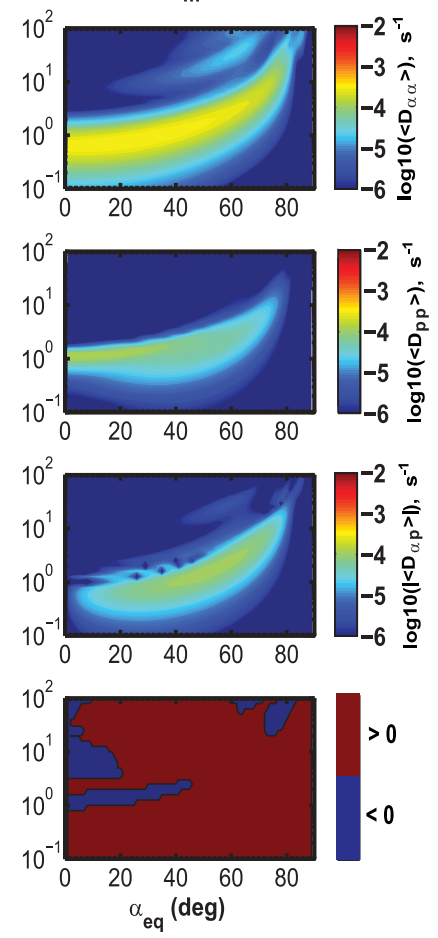

Figure 6. Same as in Figure 3, except for upper band chorus scattering corresponding to four wave normal angle distributions with peak wave normal angle $\theta_{m}$ at (a) $0^{\circ}$, (b) $20^{\circ}$, (c) $40^{\circ}$, and (d) $44^{\circ}$. 


\section{Upper-band chorus $\left(0.5<\mathrm{f} / \mathrm{f}_{\mathrm{ce}}<0.7\right)$}

(a) $300 \mathrm{eV}$
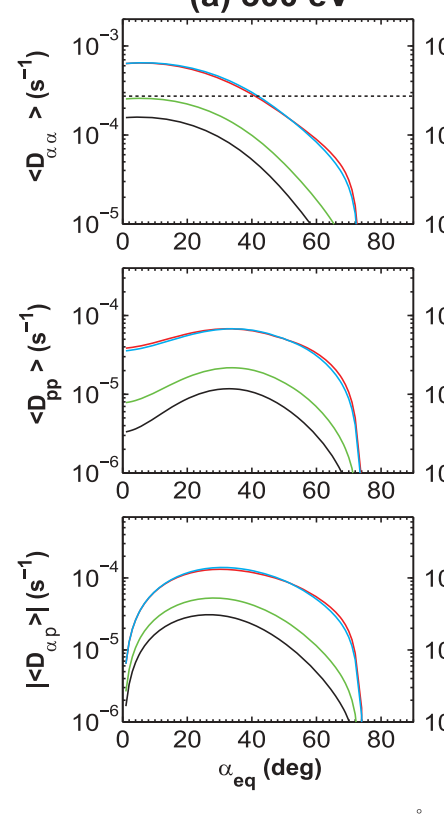

$\rightarrow \theta_{m}=0^{\circ}$ (b) $1 \mathrm{keV}$
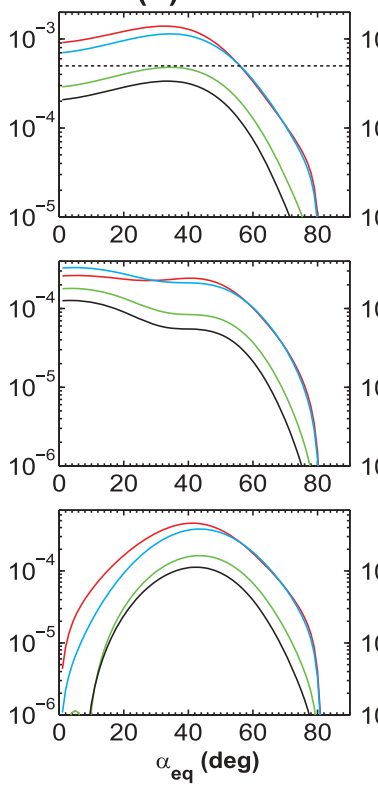

$-\theta_{m}=20^{\circ}$ (c) $3 \mathrm{keV}$
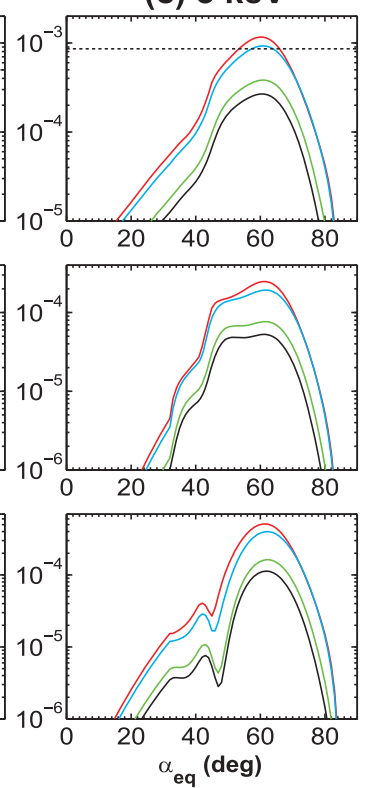

$\leftarrow \theta_{\mathrm{m}}=40^{\circ} \quad \longrightarrow \theta_{\mathrm{m}}=44^{\circ}$ (d) $10 \mathrm{keV}$
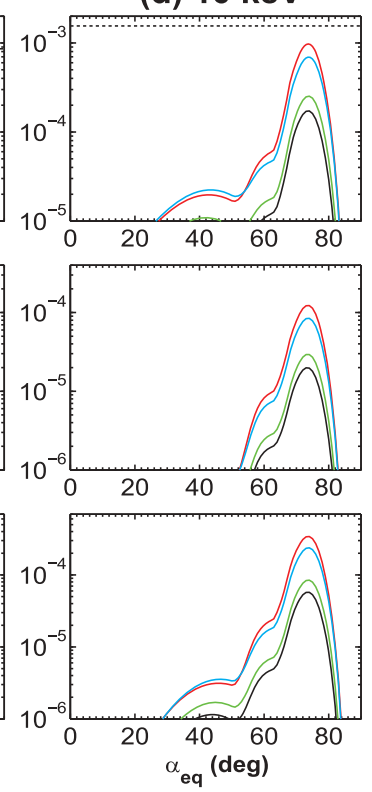

Figure 7. Comparison of upper band chorus induced bounce-averaged resonant diffusion coefficients at $L=6$ as a function of equatorial pitch angle for plasma sheet electrons at the six specified energies from $300 \mathrm{eV}$ to $10 \mathrm{keV}$ by adopting the four wave normal angle distributions peaking respectively at $0^{\circ}, 20^{\circ}$, $40^{\circ}$, and $45^{\circ}$. The horizontal dashed lines represent the strong diffusion rate $D_{S D}$.

\section{Upper-band chorus $\left(0.5<f / f_{c e}<0.7\right)$}

(a) $300 \mathrm{eV}$
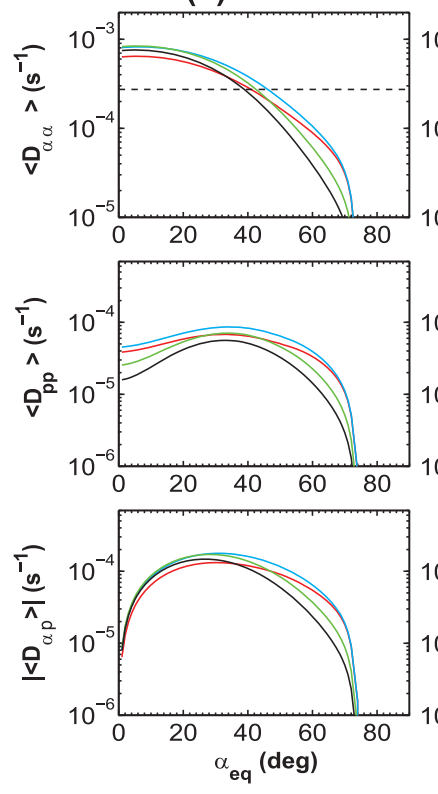

$\rightarrow \theta_{m}=0^{\circ}$ (b) $1 \mathrm{keV}$
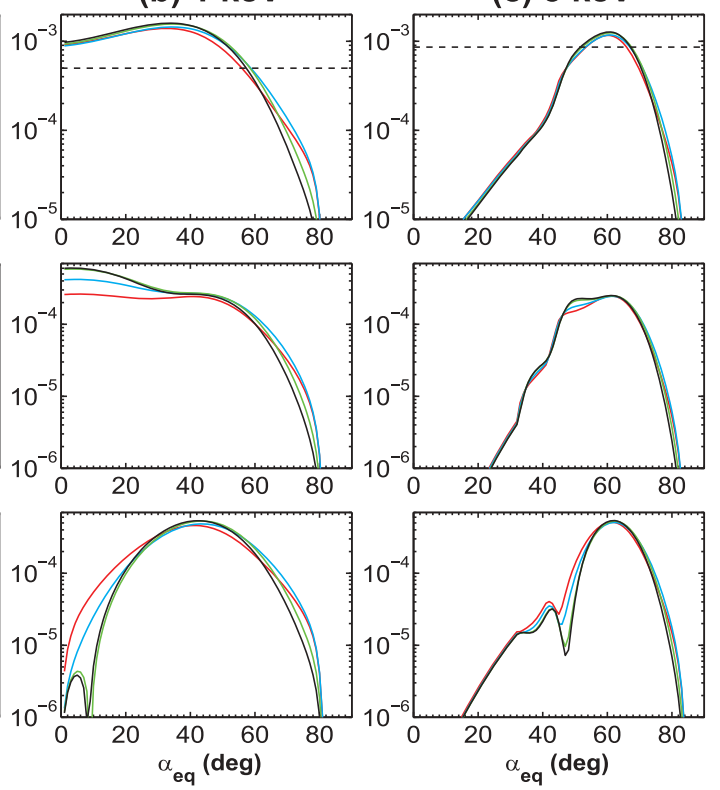

(c) $3 \mathrm{keV}$
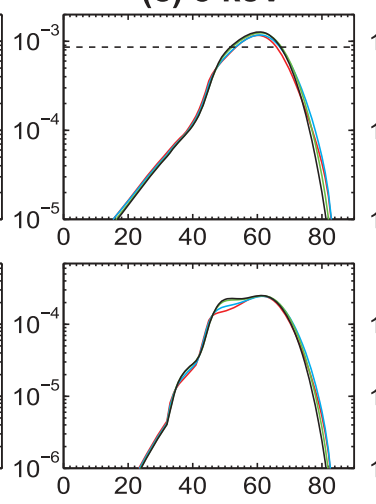

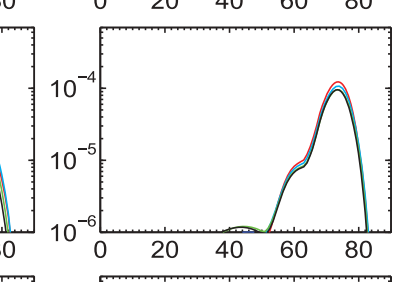

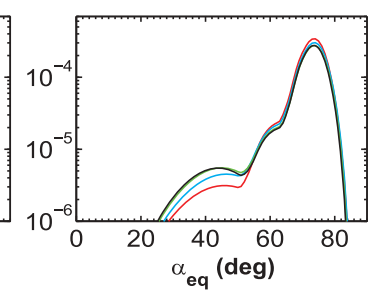

(d) $10 \mathrm{keV}$

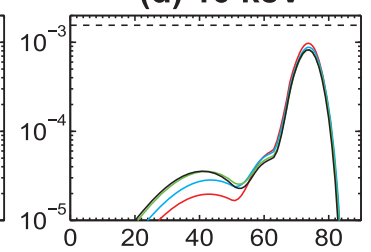

$\longrightarrow \theta_{m}=40^{\circ} \quad-\theta_{m}=44^{\circ}$

$\ldots \ldots . . \mathrm{D}_{\mathrm{SD}}$

Figure 8. Same as in Figure 7, except that the adopted upper band chorus magnetic field amplitude is the same as that for $\theta_{m}=0^{\circ}$. 
scattering on a timescale of hours near the loss cone occurs for a smaller population of plasma sheet electrons, confined to a narrower energy range from $\sim 100 \mathrm{eV}$ to $\sim 1 \mathrm{keV}$. Momentum diffusion and mixed diffusion are generally slower than pitch angle diffusion, showing variations with wave normal angle distribution in a manner similar to that for $\left\langle D_{\alpha \alpha}\right\rangle$.

[14] Figure 7 shows UBC-induced diffusion rates at $\mathrm{L}=6$ as a function of $\alpha_{e q}$ for four specified energies from $300 \mathrm{eV}$ to $10 \mathrm{keV}$ and compares $\left\langle D_{\alpha \alpha}\right\rangle$ with the strong diffusion rates $D_{s d}$ (horizontal dashed line). Changes in UBC scattering rates due to variations in wave normal angle distribution are substantial, especially when the waves propagate highly obliquely. With the same electric field spectral intensities, moderate-time UBC peaking at $\theta_{m}=0^{\circ}$ and $20^{\circ}$ can cause efficient pitch angle scattering (exceeding the strong diffusion rates) near the loss cone for $300 \mathrm{eV}$ and $1 \mathrm{keV}$ electrons. However, highly oblique waves $\left(\theta_{m}=44^{\circ}\right)$ would induce precipitation losses below the strong diffusion limit. Although UBC diffusion rates vary markedly with changes in peak wave normal angle, the curves of all three diffusion rates present similar shapes with respect to equatorial pitch angle and the coefficients show a common decreasing trend with increasing $\theta_{m}$, in a good agreement with the decrease in UBC magnetic field amplitude when $\theta_{m}$ increases. To investigate whether the variations in diffusion rates result from the changes in $B_{w}$ or from the redistribution of wave power over wave normal angle, Figure 8 shows the bounce-averaged scattering rates by UBC computed for the four different wave normal angle distributions for a fixed $B_{w}$ which we set to the value for $\theta_{m}=0^{\circ}$. The differences in scattering rates for increasing $\theta_{m}$ are minor except for $300 \mathrm{eV}$ electrons, suggesting that changes in wave magnetic field amplitude $\left(B_{w}\right)$ play a critical role in quantifying UBCdriven scattering rates and mainly account for the dependence of diffuse auroral scattering by UBC on wave normal angle distribution for given wave electric field intensities.

\section{Discussion and Conclusions}

[15] Changes in wave normal angle can alter both the refractive index $n$ and the configuration between the wave electric field $\vec{E}$ and the propagation vector $\vec{k}$ for both lower band chorus and upper band chorus. Since both LBC and UBC emissions have been reported to propagate over a broad range of angles with respect to the ambient magnetic field, the effects of changes in the wave normal angle should be included for evaluation of chorus scattering rates, particularly when using such data sets as CRRES for which only electric field intensity measurements are originally available, lacking wave normal angle information. Thus, the parallel propagation assumption has been extensively adopted to analyze the CRRES electric field wave data [e.g., Meredith et al., 2003; Ni et al., 2011a, 2011b] to construct the wave magnetic field model for quantifying the scattering rates.

[16] Our computations of bounce-averaged quasi-linear diffusion coefficients for plasma sheet electrons take into account variations in LBC and UBC wave magnetic field amplitude and frequency spectrum by adopting different peak wave normal angles for statistically averaged electric field intensities observed on CRRES around $\mathrm{L}=6$. The results show that both $\mathrm{LBC}$ and $\mathrm{UBC}$ driven diffuse auroral scattering rates, when calculated from electric field measurements, depends sensitively on wave normal angle distribution. For UBC-driven diffuse auroral scattering, there is a good agreement between the decrease in bounce-averaged diffusion coefficients and the decrease in UBC magnetic field amplitude when $\theta_{m}$ increases, suggesting that changes in wave magnetic field amplitude $\left(B_{w}\right)$ play a dominant role in controlling the scattering rates of plasma sheet electrons by UBC, which is also consistent with the finding of Shprits and $N i$ [2009]. Although the decrease in $B_{w}$ can explain the decrease in LBC scattering rates for 3-10 keV electrons at lower $\alpha_{e q}$ and for $30 \mathrm{keV}$ electrons at intermediate $\alpha_{e q}$, it cannot explain the increase in LBC scattering rates for $1 \mathrm{keV}$ at all resonant pitch angles, for 3-10 keV electrons at higher $\alpha_{e q}$, and for $30 \mathrm{keV}$ electrons at lower $\alpha_{e q}$, which is more likely due to the decrease in resonant energy and the redistribution of wave power over wave normal angle for increased peak wave normal angle. Therefore, LBC driven diffuse auroral scattering tends to respond to variation in wave normal angle distribution in a more complex manner than UBC-driven diffuse auroral scattering.

[17] Increasing $\theta_{m}$ for LBC and UBC can cause the combined (net) pitch angle scattering rates to vary from above the strong diffusion level to below it, thereby altering the loss timescales of plasma sheet electrons and affecting the intensity and MLT distribution of the diffuse aurora and the evolution of electron pitch angle distribution including the formation of electron pancake distributions [e.g., Meredith et al., 1999; Li et al., 2010; Tao et al., 2011a]. Variations in momentum diffusion and cross diffusion associated with variations in wave normal angle distribution can also modify the wave-electron energy transfer processes.

[18] Since Gaussian fits have been extensively and intensively used by numerous previous studies to describe chorus wave power spectrum, we have applied the Gaussian fits to the wave magnetic field spectral intensities converted from the CRRES electric field spectral intensities for calculations of quasi-linear diffusion rates. Our diffusion code (the UCLA Full Diffusion Code [Ni et al., 2008; Shprits and Ni, 2009]), similar to the other diffusion codes such as Lyons [1974a, 1974b], Glauert and Horne [2005], Summers et al. [2007b], Albert [2005, 2007, 2008] and Xiao et al. [2009], has implemented the standard method to compute scattering rates by defining the wave power as a Gaussian distribution as a function of frequency. While quasi-linear diffusion calculations with modeled Gaussian spectrum of chorus waves have produced a number of simulation results consistent with observations [e.g., Thorne et al., 2005, 2007, 2010; Horne et al., 2005; Albert et al., 2009; Su et al., 2009], we have performed a number of test calculations of bounce-averaged diffusion coefficients by applying linear interpolation of wave spectral intensity at resonant frequencies between adjacent observation points of wave frequency instead of using the fitted Gaussian spectrum. Comparisons between the diffusion coefficients obtained using the two different wave spectral intensity profiles (the results not shown) indicate that the scattering rates are similar to each other for both LBC and UBC when electron energy and wave normal angle distribution is fixed and that the changes in diffusion rates due to use of Gaussian spectrum are generally much smaller than those introduced by variations 
in wave normal angle distribution. A recent survey of the Poynting flux of chorus waves by Santolik et al. [2010] showed a "heavy tail" feature of chorus power spectral densities in terms of probability distributions, which can be modeled by a power law or lognormal model and suggests an alternative for chorus wave power spectrum in our following studies.

[19] In addition, we have utilized the available CRRES wave data sets which have been smoothed by using a running 3-min average to take out the beating effects due to differences in the sampling and the spin rate. As a consequence, the constructed chorus wave model is an average profile without the details of wave packets. To investigate the characteristics of chorus wave packets is outside the scope of this paper and is a very interesting topic that can be left for future studies. However, we have improved the moderate-time nightside chorus wave model by including the latitudinal dependence of wave power. Basically, we have chosen each individual wave profile in any specified magnetic latitude interval by averaging the wave intensities taken over $0.1 \mathrm{~L}$. The average profile is typically calculated from 30-100 individual wave profiles. Therefore, we have a reasonable number of wave events to perform a reliable statistical analysis. On the other hand, Li et al. [2008, 2009a], based upon linear growth theory and ray tracing simulations, have shown that the chorus wave rays propagate generally around the same magnetic field line crossing a small range of $\mathrm{L}$, typically $\Delta \mathrm{L} \approx 0.2$ with the same crossing point at the equator, before the waves are strongly attenuated due to Landau resonance once the wave normal angles exceed $30^{\circ}$ at higher latitudes $\left(|\lambda|>\sim 5^{\circ}\right)$. Those results suggest that it can be a quite good approximation to assume an extremely narrow L-shell interval for nightside chorus propagation (confined to $|\lambda| \leq 15^{\circ}$ ) and assume the random-phase interactions between many chorus wave packets and electrons. Even under the situation that the wave packets at different magnetic latitudes originate from different source locations due to the wave propagation properties, it remains feasible to apply quasi-linear formulation for bounce-averaged diffusion calculations to evaluate the average scattering effect of latitudinally distributed chorus emissions under the assumption that these waveparticle interaction processes are additive and independent. While further experimental studies are required to clarify this, the applicability of quasi-linear theory to quantifying scattering rates by combinations of chorus wave packets with small or moderate amplitude have been numerically confirmed by Albert [2010] and Tao et al. [2011b].

[20] While the presented results concentrate on $L=6$ under geomagnetically moderate conditions, our findings are appropriate to LBC- and UBC-driven diffuse auroral precipitation at other $L$-shells, within other MLT intervals, and under other geomagnetic activity levels. It is also worth noting that cold plasma theory limits whistler mode wave propagation at angles less than $\theta_{\text {res }}$, that is, in an electromagnetic nature. When chorus emissions propagate obliquely very close to $\theta_{\text {res }}$, use of the hot plasma dispersion relation is required for the computation of diffusion coefficients since the waves become quasi-electrostatic or electrostatic, which will be a subject of future research. In addition, a dipole magnetic field model has been adopted for simplicity to compute bounce-averaged quasi-linear diffu- sion coefficients. Recent studies by Orlova and Shprits [2010] and Ni et al. [2011a] have demonstrated that chorus driven electron scattering rates and the resultant loss timescales at $\mathrm{L} \geq 6$ on the nightside can significantly depend on the adopted magnetic field model, which requires further investigation to establish a more realistic model of diffuse auroral precipitation by magnetospheric chorus.

[21] Acknowledgments. This research was supported by NSF grant ATM-0802843 and NASA grant NNX09AF51G.

[22] Philippa Browning thanks the reviewers for their assistance in evaluating this paper.

\section{References}

Albert, J. M. (2005), Evaluation of quasi-linear diffusion coefficients for whistler mode waves in a plasma with arbitrary density ratio, J. Geophys. Res., 110, A03218, doi:10.1029/2004JA010844.

Albert, J. M. (2007), Simple approximations of quasi-linear diffusion coefficients, J. Geophys. Res., 112, A12202, doi:10.1029/2007JA012551.

Albert, J. M. (2008), Efficient approximations of quasi-linear diffusion coefficients in the radiation belts, J. Geophys. Res., 113, A06208, doi:10.1029/2007JA012936.

Albert, J. M. (2010), Diffusion by one wave and by many waves, J. Geophys. Res., 115, A00F05, doi:10.1029/2009JA014732.

Albert, J. M., and Y. Y. Shprits (2009), Estimates of lifetimes against pitch angle diffusion, J. Atmos. Sol. Terr. Phys., 71, 1647-1652, doi:10.1016/j. jastp.2008.07.004

Albert, J. M., N. P. Meredith, and R. B. Horne (2009), Three-dimensional diffusion simulation of outer radiation belt electrons during the 9 October 1990 magnetic storm, J. Geophys. Res., 114, A09214, doi:10.1029/ 2009JA014336.

Anderson, P. C., S. M. Petrinec, and K. Liou (2001), Statistical patterns in $\mathrm{X}$-ray and UV auroral emissions and energetic electron precipitation, J. Geophys. Res., 106(A4), 5907-5911, doi:10.1029/2000JA003041.

Anderson, R. R., D. A. Gurnett, and D. L. Odere (1992), CRRES plasma wave experiment, J. Spacecr. Rockets, 29, 570-573, doi:10.2514/ 3.25501 .

Bortnik, J., R. M. Thorne, and N. P. Meredith (2007), Modeling the propagation characteristics of chorus using CRRES suprathermal electron fluxes, J. Geophys. Res., 112, A08204, doi:10.1029/2006JA012237.

Breneman, A. W., C. A. Kletzing, J. Pickett, J. Chum, and O. Santolík (2009), J. Geophys. Res., 114, A06202, doi:10.1029/2008JA013549.

Burtis, W. J., and R. A. Helliwell (1976), Magnetospheric chorus: Occurrence patterns and normalized frequency, Planet. Space Sci., 24, 1007-1010, doi:10.1016/0032-0633(76)90119-7.

Burton, R., and R. Holzer (1974), The origin and propagation of chorus in the outer magnetosphere, J. Geophys. Res., 79(7), 1014-1023, doi:10.1029/JA079i007p01014.

Gendrin, R. (1961), Le guidage des whistlers par le champ magnetique, Planet. Space Sci., 5, 274-282, doi:10.1016/0032-0633(61)90096-4.

Glauert, S. A., and R. B. Horne (2005), Calculation of pitch-angle and energy diffusion coefficients with the PADIE code, J. Geophys. Res., 110, A04206, doi:10.1029/2004JA010851.

Goldstein, B., and B. Tsurutani (1984), Wave normal directions of chorus near the equatorial source region, J. Geophys. Res., 89(A5), 2789-2810, doi:10.1029/JA089iA05p02789.

Haque, N., M. Spasojevic, O. Santolík, and U. S. Inan (2010), Wave normal angles of magnetospheric chorus emissions observed on the Polar spacecraft, J. Geophys. Res., 115, A00F07, doi:10.1029/2009JA014717.

Hayakawa, M., Y. Yamanaka, M. Parrot, and F. Lefeuvre (1984), The wave normals of magnetospheric chorus emissions observed on board GEOS 2, J. Geophys. Res., 89(A5), 2811-2821, doi:10.1029/JA089iA05p02811.

Horne, R. B., et al. (2005), Wave acceleration of electrons in the Van Allen radiation belts, Nature, 437, 227, doi:10.1038/nature03939.

Hospodarsky, G. B., T. F. Averkamp, W. S. Kurth, D. A. Gurnett, M. Dougherty, U. Inan, and T. Wood (2001), Wave normal and Poynting vector calculations using the Cassini radio and plasma wave instrument, J. Geophys. Res., 106(A12), 30,253-30,269.

Kennel, C. F., and F. Engelmann (1966), Velocity space diffusion from weak plasma turbulence in a magnetic field, Phys. Fluids, 9, 2377-2388, doi:10.1063/1.1761629.

Kennel, C. F., and R. M. Thorne (1967), Unstable growth of unducted whistlers propagating at an angle to the geomagnetic field, J. Geophys. Res., 72(3), 871-878, doi:10.1029/JZ072i003p00871. 
Lauben, D. S., U. S. Inan, T. F. Bell, and D. A. Gurnett (2002), Source characteristics of ELF/VLF chorus, J. Geophys. Res., 107(A12), 1429, doi:10.1029/2000JA003019.

LeDocq, M. J., D. A. Gurnett, and G. B. Hospodarsky (1998), Chorus source locations from VLF Poynting flux measurements with the Polar spacecraft, Geophys. Res. Lett., 25(21), 4063-4066, doi:10.1029/ 1998GL900071.

Li, W., R. M. Thorne, N. P. Meredith, R. B. Horne, J. Bortnik, Y. Y. Shprits, and B. Ni (2008), Evaluation of whistler mode chorus amplification during an injection event observed on CRRES, J. Geophys. Res., 113, A09210, doi:10.1029/2008JA013129.

Li, W., R. M. Thorne, V. Angelopoulos, J. W. Bonnell, J. P. McFadden, C. W. Carlson, O. LeContel, A. Roux, K. H. Glassmeier, and H. U. Auster (2009a), Evaluation of whistler-mode chorus intensification on the nightside during an injection event observed on the THEMIS spacecraft, J. Geophys. Res., 114, A00C14, doi:10.1029/2008JA013554.

Li, W., et al. (2009b), Global distribution of whistler-mode chorus waves observed on the THEMIS spacecraft, Geophys. Res. Lett., 36, L09104, doi:10.1029/2009GL037595.

Li, W., et al. (2010), THEMIS analysis of observed equatorial electron distributions responsible for the chorus excitation, J. Geophys. Res., 115, A00F11, doi:10.1029/2009JA014845.

Lyons, L. R. (1974a), General relations for resonant particle diffusion in pitch angle and energy, J. Plasma Phys., 12, 45-49, doi:10.1017/ S0022377800024910.

Lyons, L. R. (1974b), Pitch angle and energy diffusion coefficients from resonant interactions with ion-cyclotron and whistler waves, J. Plasma Phys., 12, 417-432, doi:10.1017/S002237780002537X.

Meredith, N. P., A. D. Johnstone, S. Szita, R. B. Horne, and R. R. Anderson (1999), "Pancake" electron distributions in the outer radiation belts, J. Geophys. Res., 104(A6), 12,431-12,444, doi:10.1029/1998JA900083.

Meredith, N. P., R. B. Horne, A. D. Johnstone, and R. R. Anderson (2000), The temporal evolution of electron distributions and associated wave activity following substorm injections in the inner magnetosphere, J Geophys. Res., 105(A6), 12,907-12,917, doi:10.1029/2000JA900010.

Meredith, N. P., R. B. Horne, and R. R. Anderson (2001), Substorm dependence of chorus amplitudes: Implications for the acceleration of electrons to relativistic energies, J. Geophys. Res., 106(A7), 13,165-13,178, doi:10.1029/2000JA900156.

Meredith, N. P., M. Cain, R. B. Horne, R. M. Thorne, D. Summers, and R. R. Anderson (2003), Evidence for chorus-driven electron acceleration to relativistic energies from a survey of geomagnetically disturbed periods, J. Geophys. Res., 108(A6), 1248, doi:10.1029/2002JA009764.

Meredith, N. P., R. B. Horne, R. M. Thorne, and R. R. Anderson (2009), Survey of upper band chorus and ECH waves: Implications for the diffuse aurora, J. Geophys. Res., 114, A07218, doi:10.1029/2009JA014230.

Muto, H., M. Hayakawa, M. Parrot, and F. Lefeuvre (1987), Direction finding of half-gyrofrequency VLF emissions in the off-equatorial region of the magnetosphere and their generation and propagation, J. Geophys. Res., 92(A7), 7538-7550, doi:10.1029/JA092iA07p07538.

Newell, P. T., T. Sotirelis, and S. Wing (2009), Diffuse, monoenergetic, and broadband aurora: The global precipitation budget, J. Geophys. Res., 114, A09207, doi:10.1029/2009JA014326.

Newell, P. T., T. Sotirelis, and S. Wing (2010), Seasonal variations in diffuse, monoenergetic, and broadband aurora, J. Geophys. Res., 115, A03216, doi:10.1029/2009JA014805.

Ni, B., R. M. Thorne, Y. Y. Shprits, and J. Bortnik (2008), Resonant scattering of plasma sheet electrons by whistler-mode chorus: Contribution to diffuse auroral precipitation, Geophys. Res. Lett., 35, L11106, doi:10.1029/2008GL034032.

Ni, B., R. M. Thorne, Y. Y. Shprits, K. Orlova, and N. P. Meredith (2011a), Chorus-driven resonant scattering of diffuse auroral electrons in nondipolar magnetic fields, J. Geophys. Res., 116, A06225, doi:10.1029/ 2011JA016453.

Ni, B., R. M. Thorne, N. P. Meredith, R. B. Horne, and Y. Y. Shprits (2011b), Resonant scattering of plasma sheet electrons leading to diffuse auroral precipitation: 2. Evaluation for whistler mode chorus waves, J. Geophys. Res., 116, A04219, doi:10.1029/2010JA016233.

Orlova, K. G., and Y. Y. Shprits (2010), Dependence of pitch-angle scattering rates and loss timescales on the magnetic field model, Geophys. Res. Lett., 37, L05105, doi:10.1029/2009GL041639.

Petrinec, S. M., D. L. Chenette, J. Mobilia, M. A. Rinaldi, and W. L. Imhof (1999), Statistical X ray auroral emissions: PIXIE observations, Geophys. Res. Lett., 26(11), 1565-1568, doi:10.1029/1999GL900295.

Santolík, O., D. A. Gurnett, J. S. Pickett, M. Parrot, and N. CornilleauWehrlin (2003), Spatio-temporal structure of storm-time chorus, J. Geophys. Res., 108(A7), 1278, doi:10.1029/2002JA009791.

Santolík, O., D. A. Gurnett, J. S. Pickett, J. Chum, and N. CornilleauWehrlin (2009), Oblique propagation of whistler mode waves in the chorus source region, J. Geophys. Res., 114, A00F03, doi:10.1029/ 2009JA014586.

Santolík, O., J. S. Pickett, D. A. Gurnett, J. D. Menietti, B. T. Tsurutani, and O. Verkhoglyadova (2010), Survey of Poynting flux of whistler mode chorus in the outer zone, J. Geophys. Res., 115, A00F13, doi:10.1029/2009JA014925.

Schulz, M., and L. Lanzerotti (1974), Particle Diffusion in the Radiation Belts, Springer, New York.

Shprits, Y. Y., and B. Ni (2009), Dependence of the quasi-linear scattering rates on the wave normal distribution of chorus waves, J. Geophys. Res. 114, A11205, doi:10.1029/2009JA014223.

Shprits, Y. Y., R. M. Thorne, R. B. Horne, and D. Summers (2006a), Bounce-averaged diffusion coefficients for field-aligned chorus waves, J. Geophys. Res., 111, A10225, doi:10.1029/2006JA011725.

Shprits, Y. Y., W. Li, and R. M. Thorne (2006b), Controlling effect of the pitch angle scattering rates near the edge of the loss cone on electron lifetimes, J. Geophys. Res., 111, A12206, doi:10.1029/2006JA011758.

Shprits, Y. Y., N. P. Meredith, and R. M. Thorne (2007), Parameterization of radiation belt electron loss timescales due to interactions with chorus waves, Geophys. Res. Lett., 34, L11110, doi:10.1029/2006GL029050.

Stix, T. H. (1962), The Theory of Plasma Waves, McGraw-Hill, New York. $\mathrm{Su}, \mathrm{Z}$., H. Zheng, and S. Wang (2009), Evolution of electron pitch angle distribution due to interactions with whistler mode chorus following substorm injections, J. Geophys. Res., 114, A08202, doi:10.1029/ 2009JA014269.

Su, Z., H. Zheng, and S. Wang (2010), A parametric study on the diffuse auroral precipitation by resonant interaction with whistler mode chorus, J. Geophys. Res., 115, A05219, doi:10.1029/2009JA014759.

Summers, D. (2005), Quasi-linear diffusion coefficients for field-aligned electromagnetic waves with applications to the magnetosphere, J. Geophys. Res., 110, A08213, doi:10.1029/2005JA011159.

Summers, D., and B. Ni (2008), Effects of latitudinal distributions of particle density and wave power on cyclotron resonant diffusion rates of radiation belt electrons, Earth Planets Space, 60, 763-771.

Summers, D., and R. M. Thorne (2003), Relativistic electron pitch-angle scattering by electromagnetic ion cyclotron waves during geomagnetic storms, J. Geophys. Res., 108(A4), 1143, doi:10.1029/2002JA009489.

Summers, D., B. Ni, and N. P. Meredith (2007a), Timescales for radiation belt electron acceleration and loss due to resonant wave-particle interactions: 1. Theory, J. Geophys. Res., 112, A04206, doi:10.1029/ 2006JA011801.

Summers, D., B. Ni, and N. P. Meredith (2007b), Timescales for radiation belt electron acceleration and loss due to resonant wave-particle interactions: 2. Evaluation for VLF chorus, ELF hiss, and electromagnetic ion cyclotron waves, J. Geophys. Res., 112, A04207, doi:10.1029/ 2006JA011993.

Tao, X., R. M. Thorne, W. Li, B. Ni, N. P. Meredith, and R. B. Horne (2011a), Evolution of electron pitch angle distributions following injection from the plasma sheet, J. Geophys. Res., 116, A04229, doi:10.1029/ 2010JA016245

Tao, X., J. Bortnik, J. M. Albert, K. Liu, and R. M. Thorne (2011b), Comparison of quasilinear diffusion coefficients for parallel propagating whistler mode waves with test particle simulations, Geophys. Res. Lett., 38, L06105, doi:10.1029/2011GL046787.

Thorne, R. M., and C. F. Kennel (1967), Quasi-trapped VLF propagation in the outer magnetosphere, J. Geophys. Res., 72(3), 857-870, doi:10.1029/ JZ072i003p00857.

Thorne, R. M., T. P. O'Brien, Y. Y. Shprits, D. Summers, and R. B. Horne (2005), Timescale for MeV electron microburst loss during geomagnetic storms, J. Geophys. Res., 110, A09202, doi:10.1029/2004JA010882.

Thorne, R. M., Y. Y. Shprits, N. P. Meredith, R. B. Horne, W. Li, and L. R. Lyons (2007), Refilling of the slot region between the inner and outer electron radiation belts during geomagnetic storms, J. Geophys. Res., 112, A06203, doi:10.1029/2006JA012176.

Thorne, R. M., B. Ni, X. Tao, R. B. Horne, and N. P. Meredith (2010), Scattering by chorus waves as the dominant cause of diffuse auroral precipitation, Nature, 467, 943-946, doi:10.1038/nature09467.

Xiao, F., Z. Su, H. Zheng, and S. Wang (2009), Modeling of outer radiation belt electrons by multidimensional diffusion process, J. Geophys. Res. 114, A03201, doi:10.1029/2008JA013580.

R. B. Horne and N. P. Meredith, British Antarctic Survey, Natural Environment Research Council, Cambridge CB3 0ET, UK.

B. Ni and M. Thorne, Department of Atmospheric and Oceanic Sciences, University of California, Los Angeles, CA 90095-1565, USA. (bbni@ atmos.ucla.edu)

Y. Y. Shprits, Institute of Geophysics and Planetary Physics, University of California, Los Angeles, CA 90095-1565, USA. 